\title{
The Effects of Traditional Neuromuscular Training and Rider Specific Training on Novice Horse-Rider Position
}

\author{
Eleanor Boden ${ }^{1}$ and Hayley Randle ${ }^{2}$ \\ 1. Department of Architecture, Construction and Environment, University of Central Lancashire in partnership with Myerscough \\ College, Bilsborrow, Preston PR3 ORY, England
}

2. Department of Animal Science, Plymouth University in partnership with Duchy College, Stoke Climsland, Callington, Cornwall PL17 8PB, England

\begin{abstract}
The equine industry and the competition rider community are increasingly acknowledging that rider strength and fitness contribute to successful performance, and other sporting disciplines are more aware of the potential to improve performance. This study aimed to determine if the rider could maintain the classical position (i.e., with a straight line between the ear, shoulder, hip and heel) pre and post an additional dismounted fitness training programme. Students $(N=16)$ completed a dismounted training programme. All candidates were self-reported as sedentary and graded "novice" by a qualified riding instructor. Initial measurements of the deviation from the ESHH (ear, shoulder, hip, heel) alignment were carried out prior to the start of the regime, weekly and at the end of the 8-week training programme. An initial data collection was carried out (prior to any additional fitness regime) in walk, rising trot and sitting trot. An exercise session was timetabled into the syllabus as well as an hour riding session to film the candidates on a weekly basis. Each rider had 3D spherical markers attached at specific anatomical landmarks; Quintic ${ }^{\text {TM }}$ Sports Biomechanics Video Analysis Software package allowed a vertical line to be drawn; the deviation of each marker from this line was recorded. Deviations from ESHH alignment were not normally distributed. The deviation of the marker in relation to time, marker placement, gait and repeat stride were tested using a Friedman test. The comparison between rider (subject) and marker (factor) was analysed using a Kruskal Wallis test. Additionally, dismounted exercise regimes suggested a significant improvement on novice rider position $(P<0.005)$, each gait had a significant effect on the deviation from the ESHH alignment $\left(N=16, \mathrm{H}_{2}=221.53 ; P<0.001\right)$. The results suggest that additional exercise programs are effective at improving novice horse rider position, however, which exercise programs are most effective in advancing performance is less understood. Personal participation was identified as a major contributing factor in improving novice rider position and therefore assists in our understanding the role of exercise. These findings suggest a role for additional team exercises in promoting fitness and improvement of mounted rider position in novice equine athletes.
\end{abstract}

Key words: Equine, fitness, student, ball sports, personal participation.

\section{Introduction}

\subsection{The Correct Rider Position}

The equine industry, particularly the competition rider community is increasingly acknowledging that rider strength and fitness is vital in achieving the best results in competition. The BHS (British Horse Society) [1] describes the correct rider position as "There are two straight lines that can be observed from the ground. The first of these is from the rider's ear, through the

Corresponding author: Eleanor Boden, M.S., research field: rider performance. E-mail: eboden@myerscough.ac.uk. shoulder and hip and his heel" (Fig. A1).

The sport of horse riding is described as unique in its posture and motion patterns, and cannot be easily compared to the actions of any other athletic endeavour. Several factors cause a horse to not move correctly, most commonly an unbalanced rider. The BHS describes the "correct" influence of the rider on the mounted horse as "independent and able to regulate energy created by the legs, in order to control the movement of the horse". This description highlights the level of control the mounted horse rider possesses over the way that the horse moves. It has been 
suggested that a rider who has correctly prepared themselves for horse riding by undertaking a proper fitness regime is able to achieve more accurate application of the instructions given to the horse whilst in the saddle $[2,3]$.

When the ESHH (ear, shoulder, hip and heel) line is maintained, maximal balance and harmony can be achieved (in theory). Schils et al. [4] demonstrated that it is possible to maintain this ESHH alignment whilst in motion as well as stating that the more advanced the rider is, the easier it is to maintain the ESHH alignment.

\subsection{Rider Performance in Relation to Fitness}

Other sports are more aware of the potential of the utilisation of additional methods to improve performance, for example, Pilates is commonly used to develop optimal strength, co-ordination and balance in modern dance and ballet. There has been a limited amount of research into rider position, possibly due to difficulties in dealing with large amount of variables associated with both the horse and the rider. However, it is known that horse riders need stamina in order to meet the intense muscular demands that are needed to maintain the correct riding posture. The importance of having strong core abdominal muscles with all of the main muscle groups working together is widely acknowledged to enable the rider to remain stable and upright [5-7].

\subsection{Rider Fitness}

Rider fitness has been associated with injury and underperformance in both the horse and rider [7-9] supporting the suggestion that improved rider fitness leads to an improvement in equine performance.

Suitable training techniques for equine students ideally will involve physically and technically demanding activity that requires the use of all muscle groups; such as rowing, the use of elliptical trainers and skipping. One of the fundamental challenges for horse riders, especially equine students, is finding time to incorporate fitness into the daily routine due to the course workload and/or other priorities [10, 11]. Hewitt et al. [12] trialed this approach at the Goucher School of Dance, where students have the opportunity to enroll in a Pilates course for academic marks which ultimately contributes to the final grade awarded for the course.

The lack of time in the busy schedules of horse riders suggests that there is a lack of appreciation of the importance of rider fitness which often results in riders not prioritising their own fitness amongst other daily tasks; hence the reason for implementing fitness into the syllabus of students within an equine college environment. The equine student does not need to be training for high speed bursts of energy, but to maintain stamina and performance for the length of the hour ride session. The exercise regimes that the equine student requires are regular controlled exercise to achieve maximal benefits as well as improve the welfare of the horse.

A fundamental problem exists when researching horse rider position is the subjective nature of scoring a horse riders position [7] and overall posture. It has been observed that a more rigid horse riding position will cause a horse to hold the head high, with the neck tensed often resulting in stiffness in the (horse's) movement [1, 13]; although the coupling between horse and rider has not been fully investigated; a well ridden horse should be as balanced as a free schooled horse [14].

\subsection{The Equine Gait}

The trot gait is commonly used in scientific studies of rider symmetry as its physical dynamics allow flaws to be exhibited (Fig. 1). It is important for the dynamics of the gaits to be fully understood; since the different stride patterns of each gait require a different sequence from the riders' position.

This study aimed to determine if the rider could maintain the classical position (i.e., with a straight line between the ear, shoulder, hip and heel) pre and post an 


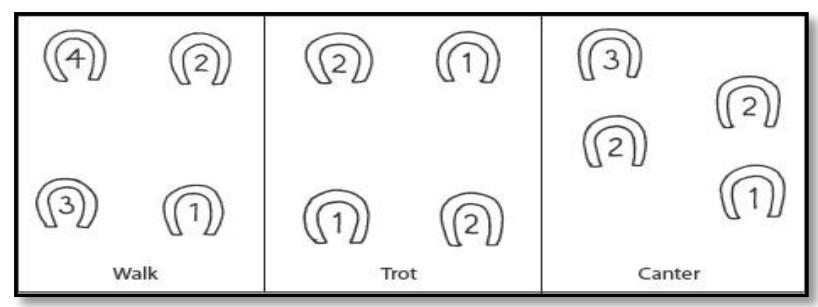

Fig. 1 Sequence of foot falls exhibited by the horse in walk, trot and canter on the left rein (i.e., when travelling anti-clockwise).

additional dismounted fitness training programme.

\section{Methods}

\subsection{Rider Characteristics}

Further education, equine students $(n=16$, Table 1$)$ were recruited for this study. Students were selected by qualified riding instructors who identified each candidate as "novice" and grouped based on skill to act as control for bias factors [15-18]. All students were expected to attend previously scheduled fitness training sessions in their weekly timetable for the January-April 2011 Term. All candidates were free of any predisposing issues after completing a PARQ (physical activity readiness questionnaire) and self-reported as "sedentary" (i.e., engaged in less than 1 hour of exercise a week) as defined by Owen et al. [19]. All candidates had previous horse riding experience "graded" as novice by qualified riding instructors. The candidates $(n=16)$ were randomly divided into two treatment groups, rider specific training $(n=7)$ and traditional neuromuscular training $(n=9)$ and completed the allocated training regime for 8 weeks and 5 weeks, respectively. The treatment groups were pre-determined by the timetabled riding sessions. Statistical analysis was carried out to ensure no significant $(P>0.05)$ difference was found in rider position between subjects in the different groups before starting the study ensuring that the study was starting with a balanced sample population to eliminate bias.

\subsection{Rider Specific Training}

Treatment 1 (rider specific) was based upon the theory that dismounted exercises are able to improve mounted rider position resulting in being more efficient in the saddle; the fundamental function of the theory is to create the ultimate workout regime that targets specific areas of the horse rider that more conventional sports do not take into consideration due to the nature of the activities. The focus of the routines designed is to create the optimal coordinative conditions to be transferable into riding whilst addressing common areas of weakness in the horse rider. The American College of Sports medicine has suggested that physical exercise should be carried out in an intermittent manner contradicting the suggestions of Chen et al. [16] who suggested that exercise should be carried out in a regular fashion especially in young people to encourage sustained participation. The weekly sessions were carried out in a circuit training situation, with a 5-minute session at each station of skipping, rider specific stretches, low intensity step exercises, rowing and elliptical training; the circuit was completed twice in a session.

\subsection{Traditional Neuromuscular Training}

Treatment group 2 (traditional neuromuscular training) involved the candidates would follow the same warm up procedure consisting of consistent running exercise, around the games field, for 10 minutes. The sport that was going to be played remained undisclosed until the 10-minute warm up had been completed. The format of which the sessions were run can be seen in Table 2. The sessions lasted an hour and were structured to incorporate time to allow familiarisation with the type of ball, mechanics of the throw/kick as well as increase the heart rate through exercises such as throwing the ball and following the pass.

\subsection{Data Collection}

The candidates ( $n=16)$ were filmed on a weekly basis, during a timetabled session, on a familiar horse in a $50 \mathrm{~m}$ long by $20 \mathrm{~m}$ wide arena (Fig. 2) on an outdoor prepared rubber fibre and sand mix surface. 
Table 1 Rider characteristics $(n=16)$.

\begin{tabular}{ll}
\hline Variable & Mean \pm SD \\
\hline Age (years) & $16.93 \pm 1.2$ \\
Height $(\mathrm{cm})$ & $158.55 \pm 5.54$ \\
Body mass $(\mathrm{kg})$ & $58.69 \pm 10.10$ \\
\hline
\end{tabular}

Table 2 The (weekly) TNT (traditional neuromuscular training) training schedule over the 5 weeks.

\begin{tabular}{ll}
\hline Week & Sport \\
\hline 1 & Handball \\
2 & Football \\
3 & Touch rugby \\
4 & Basketball \\
5 & Handball \\
\hline
\end{tabular}

The riders were asked to ride a horse that they had ridden before. The participants were instructed to ride down a $6 \mathrm{~m}$ wide track, defined by show jumping poles, situation on either side of the centre line to allow films to be captured. The track way ( $2 \mathrm{~m}$ width) was set down the centre of the school (centre line) to avoid any preference of rein direction which could impact on the horses and subsequently the riders' position.

\subsection{Marker Placement}

The researcher attached $60 \mathrm{~mm}$ spherical polystyrene markers attached with semi-permanent double-sided adhesive pads to anatomical landmarks down the right hand side of the body as seen in Fig. A2. The pads remained on the sweatshirts, jodhpurs and boots throughout the whole 8 weeks data collection period to prevent any inaccuracies in repeat marker placements. Anatomical landmarks were identified by palpitation prior to marker attachment at the commencement of the study. The shoulder (glenohumeral joint centre), hip (greater troncanter of the femur) and heel of the riding boot adapted from Lagarde et al. [20]. The markers enable an accurate measurement of deviation from the ESHH alignment.

Measurements of any deviations from the ESHH alignment were taken whilst subjects were mounted on horseback, when riding in walk, sitting trot and rising trot before any additional training took place. The data collection procedure was then repeated on a weekly basis until the completion of the fitness programmes.

\subsection{Fitness Testing}

A final data collection session was filmed upon conclusion of the additional treatments. In addition to the mounted measurements, fitness data were collected using the $20 \mathrm{~m}$ shuttle run test, the sit and reach test and resting heart rate before and after additional dismounted exercise.

\subsection{Data Analysis}

Using Quintic ${ }^{\mathrm{TM}}$ biomechanical software, the videos that had been collected were validated using a meter stick in every captured stride reducing any possible areas for error in the data sampling. The first stride and the last stride were discarded to reduce the potential impact of the horse turning down the guided track may have imposed on the rider position. In all paces, the point at which the video was stopped and the measurement taken was when the off fore was in full stance phase (fetlock perpendicular to the ground).

A vertical line was drawn using the automatic drawing tool by selecting the centre point of the hip marker. This vertical line enabled an imaginary ESHH

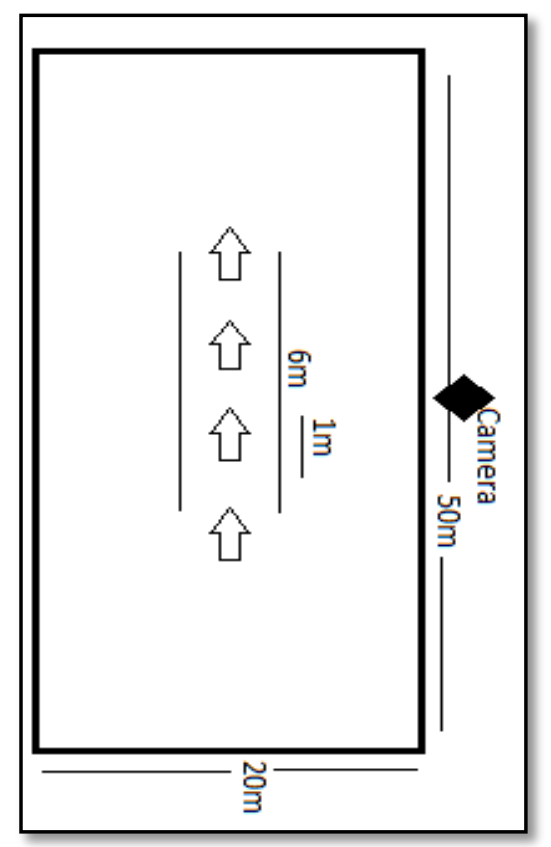

Fig. 2 Arena set up during data collection sessions. 
alignment to be visualised and the deviations can be measured. The measuring tool in the Quintic ${ }^{\mathrm{TM}}$ software package was used to find the centre of each marker to enable a more accurate placement of measurements.

\subsection{Statistical Analysis}

All data were collated using MS Excel software and then tested for normality of distribution using an Anderson Darling test in the Minitab ${ }^{\mathrm{TM}}$ v16. If a normal distribution was present, a paired t test was used to determine the extent of differences in all fitness tests and leg length pre- and post-fitness treatments. Deviations from ESHH alignment were not normally distributed. The deviation of the marker in relation to time, marker placement, gait and repeat stride were tested using a Friedman test. The comparison between rider (subject) and marker (factor) were analysed using a Kruskal-Wallis test. For all statistical tests, a probability of $P<0.05$ alpha was used

\section{Results}

\subsection{Effect of Gait on ESHH Alignment}

Each gait had a significant effect on the deviation from the ESHH alignment $\left(n=16, \mathrm{H}_{2}=221.53 ; P<\right.$ $0.001)$. Both treatment groups demonstrated a similar significant effect $\left(n=7, \mathrm{H}_{2}=133.25 ; P<0.001, n=9\right.$, $\mathrm{H}_{2}=101.75 ; P<0.001$, respectively) (Fig. 3). Both rider specific training showed significant improvement
(Fig. 3) in rider position $(P<0.005)$.

\subsection{Impact of Treatment Group}

Engagement in additional dismounted exercise, either traditional neuromuscular training or rider specific training methods did not result in a significant difference in RHR (resting heart rate) $\left(\mathrm{t}_{7}=3.18 ; P>\right.$ $0.05)$ or BT (bleep test) $\left(\mathrm{t}_{1}=2.0 ; P>0.05\right)$ in the rider specific training group. However, significant differences between RHR and BT were seen in the traditional neuromuscular training group $\left(\mathrm{t}_{5}=6.15 ; P<\right.$ 0.001 and $\mathrm{t}_{4}=11.83 ; P<0.05$ respectively, see Tables 3 and 4).

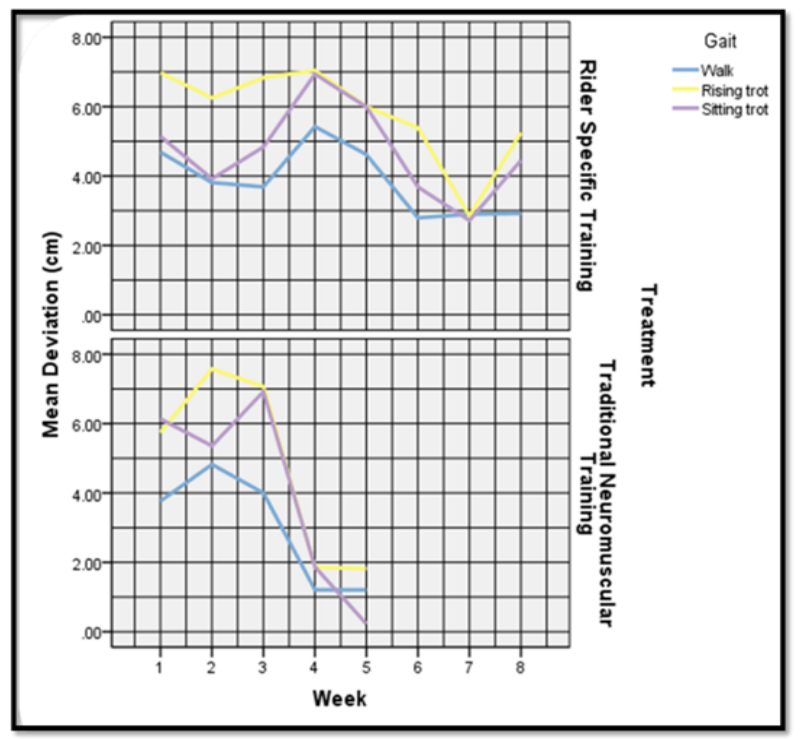

Fig. 3 The effect of RST (rider specific training) and TNT (traditional neuromuscular training)groups in relation to mean deviation $(\mathrm{cm})$ during walk, rising trot and sitting trot.

Table 3 Fitness results for TNT (Traditional Neuromuscular training).

\begin{tabular}{lllllll}
\hline Candidate & \multicolumn{2}{l}{ Resting heart rate $(\mathrm{bpm})$} & \multicolumn{3}{l}{ Sit and reach $(\mathrm{cm})$} & \multicolumn{3}{l}{ Bleep test (level) } \\
\cline { 2 - 7 } & Before & After & Before & After & Before & After \\
\hline 1 & $89 \pm 9$ & $62 \pm 6$ & 25 & 28 & 3.1 & 5.1 \\
2 & $83 \pm 5$ & $65 \pm 5$ & 24 & 30 & 5 & 7.5 \\
3 & $76 \pm 4$ & $65 \pm 4$ & 10 & 16 & 4.2 & 8 \\
4 & $80 \pm 12$ & $66 \pm 3$ & 27 & 29 & 2.2 & 5.1 \\
5 & $92 \pm 5$ & $61 \pm 7$ & 27 & 28 & 3.5 & 5.6 \\
6 & $89 \pm 1$ & $65 \pm 4$ & 3 & 5 & 3 & 5 \\
7 & $81 \pm 5$ & $69 \pm 3$ & 10 & 25 & 3.5 & 6.1 \\
8 & $86 \pm 1$ & $61 \pm 3$ & 20 & 15 & 4 & 6 \\
9 & $72 \pm 6$ & $60 \pm 4$ & 25 & 25 & 6 & 9.1 \\
\hline
\end{tabular}

Mean and standard deviation values of fitness results of the RST before and after the completion of additional training regime. Significance is shown in text. 
Table 4 Fitness results for RST (Rider Specific training group).

\begin{tabular}{|c|c|c|c|c|c|c|}
\hline \multirow[t]{2}{*}{ Candidate } & \multicolumn{2}{|c|}{ Resting heart rate (bpm) } & \multicolumn{2}{|c|}{ Sit and reach $(\mathrm{cm})$} & \multicolumn{2}{|c|}{ Bleep test (level) } \\
\hline & Before & After & Before & After & Before & After \\
\hline 1 & $91 \pm 1$ & $70 \pm 2$ & 19 & 23 & 4 & 5 \\
\hline 2 & $80 \pm 2$ & $74 \pm 2$ & 24 & 35 & 5 & 5.4 \\
\hline 3 & $92 \pm 1$ & $75 \pm 5$ & 25 & 28 & 4 & 4 \\
\hline 4 & $67 \pm 2$ & $70 \pm 8$ & 19 & 17 & 5 & 5.2 \\
\hline 5 & $65 \pm 2$ & $66 \pm 3$ & 17 & 22 & 4 & 5.2 \\
\hline 6 & $70 \pm 1$ & $61 \pm 1$ & 19 & 22 & 5.4 & 8 \\
\hline 7 & $65 \pm 2$ & $66 \pm 7$ & 24 & 35 & 6.2 & 7 \\
\hline
\end{tabular}

Mean and standard deviation values of fitness results of the TNT before and after the completion of the additional training regime.

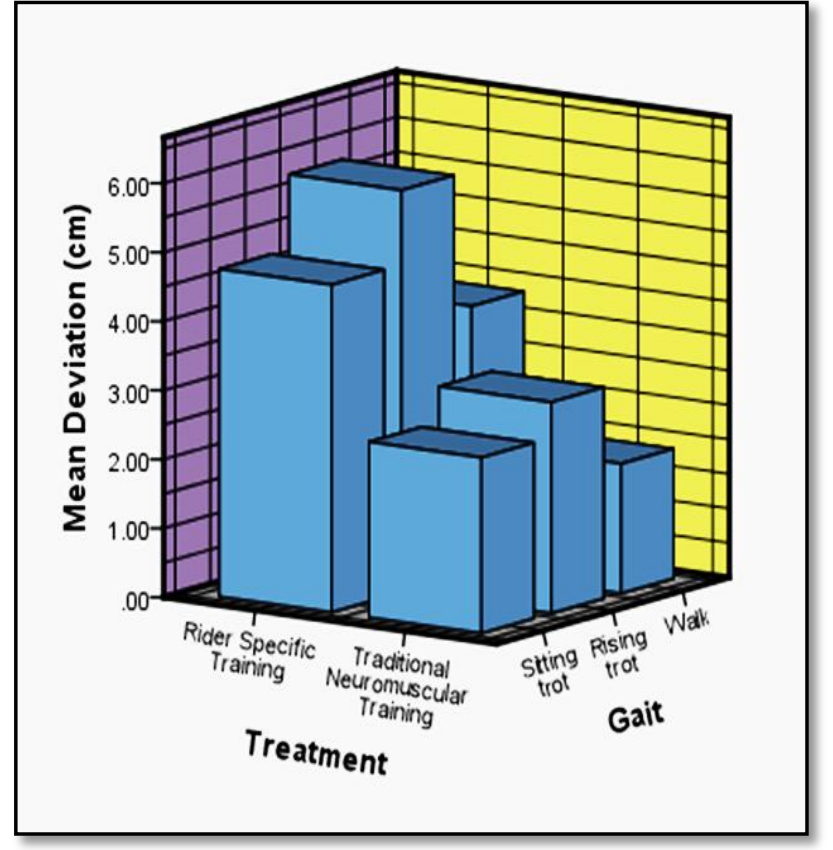

Fig. 4 Impact of gait, training TNT $(n=7)$ and RST $(n=9)$ on deviation $(\mathrm{cm})$ from ESHH alignment. Bars indicate the mean deviation from ESHH alignment in relation to marker position for three consecutive repeat observations are shown in sample of 16 riders who have undertaken additional training regimes.

The sit and reach test suggested that there was a significant difference in the rider specific training group $(9.17 \pm 3.05)\left(t_{4}=10.55 ; P<0.05\right)$ in comparison to no significant changes $\left(t_{2}=2.55 ; P>\right.$ $0.05)$ in the traditional neuromuscular training $(6.3 \pm$ $1.5 \mathrm{~cm})$, as well improvements in the traditional neuromuscular training treatment $(P<0.005)$.

The deviation of markers (Fig. 4) in the rider specific training $\left(n=7, \mathrm{H}_{2}=351.38 ; P<0.001\right)$ displayed a significant difference of each markers deviation in line with the findings of the ball sports $\left(n=9, \mathrm{H}_{2}=377.48\right.$;
$P<0.001)$

\section{Discussion}

The results of this study were consistent with the findings of Hewitt et al. [12] who concluded that a programme of complimentary, dismounted exercises can improve balance, muscular endurance and improved proprioceptive awareness in novice riders. It is widely believed that horse riders should be upright and balanced to enable accurate interactions between horse and rider whilst performing even the most simple of movements. However, the exact physiological demands on the horse rider remain unknown. The purpose of this study was to examine the efficiency of two different fitness treatments on novice horse riders. Consistent with the hypothesis, all riders showed regular decreased deviations from the ESHH alignment with the use of complementary fitness regimes. The current study demonstrates that a rider specific training regime (treatment 1) can lead to significant improvements over the course of 8 weeks in novice riders. Interestingly, however, the candidates that took part in the 8-week rider specific training regimen did not show significant improvements in the $20 \mathrm{~m}$ shuttle run although did show a significant improvement in the sit and reach test measurements. This lack of improvement on an endurance related measure could possibly be due to the absence of exertion and increase in heart rate required during the rider specific regime. The exercises required during RST were low intensity with a high repetition rate to a deliberately planned system to enable optimal results to be produced $[1,16]$. 
There has been limited research into the optimum length of any fitness regimen to gain maximal results in mounted performance. This study suggests that weekly sessions of intensive traditional neuromuscular training over 5 weeks can improve the ESHH alignment. The reduction in deviation cannot be seen until the second week in sitting trot and the third week for walk and rising trot. The sitting trot being the initial gait that shows improvement at the second week is surprising as this is the most difficult position for a rider to maintain $[1,16,21]$. Furthermore, the different treatment groups were led by different coaches. Due to the nature of the sessions and activities, the leadership requirements and styles were different; the neuromuscular-ball sports group were led by a sports coaching lecturer which had experience in working in an elite environment from a professional view point and promoted engagement through a personal passion for sport. Nielsen et al. [18], Bray et al. [22] and Thorell et al. [23] maintained that enthusiasm is easily promoted to individuals in a team situation; this may not have been the case in this study for the rider specific training treatment group.

Limitations of the study design included the absence of a control group. It is important to note that this research was carried out in a working college environment which required scrupulous ethics examination to ensure that the study would not impede the progress of the students resulting in a limited amount of criticisms which were out of the researcher's control. Due to the nature of the study, it was not possible to co-ordinate a separate control group due to student availability for participation. Each candidate was used as their own control by taking measurements (before and after) in each pace and of all fitness markers $[12,24,25]$. An unexpected aspect which was not addressed prior to the data collection was the mental preparation required before training or preparing to ride. The "desire" or "want" to succeed or improve has been highlighted through this research. It is felt that the results shown could have been affected by the attitude of the candidates during the fitness session $[22,26,27]$. It was not possible to quantify if the candidate was putting enough effort into each movement, stretch or activity.

\section{Conclusion}

Additional dismounted exercise significantly $(P<$ $0.05)$ improves mounted rider position. It is reasonable to assume that the relationship between the treatment and decrease in deviation from the ESHH alignment is due to the general implementation of an additional exercise regime. However, the traditional neuromuscular training treatment group showed significant improvements at a quicker rate than the rider specific training group. The impact of personal participation was identified as a major contributing factor in using dismounted exercise in improving novice rider position and therefore assists in our understanding the role of exercise that could be most beneficial to further education equine students. Even though this study was undertaken on a relatively small sample of further education students, these findings suggest a role for additional team exercises in promoting fitness and improvement of mounted rider position in novice equine athletes and ultimately improving rider safety and horse welfare.

\section{References}

[1] Ahearn, E. L. 2011. "The Pilates Method and Ballet Technique: Applications in the Dance Studio." Journal of Dance Education 6 (3): 92-9.

[2] Blokhuis, M. 2010. "Practical Application of Dismounted Exercises to Improve the Riders Seat." In Proceedings of International Equitation Science, Sweden.

[3] Schils, S., Greer, L., Stoner, L., and Kobluk, N. 1993. "Kinematic Analysis of the Equestrian-Walk, Posting Trot and Sitting Trot." Human Movement Science 12 (6): 693-712.

[4] de Cocq, P., van Weeren, P., and Back, W. 2004. "Saddle Pressure Measuring: Validity, Reliability and Power to Discriminate between Different Saddle-Fits." The Veterinary Journal 172 (2): 265-73.

[5] Carolien C. B. M. M., Kathalijne, E. K. V., Jan van den, B., Marianne, M. S. O. 2012. "The Influence of Challenging Objects and Horse-Rider Matching on Heart Rate, Heart Rate Variability and Behavioural Score in Riding Horses." 
The Veterinary Journal 192 (1): 75-80.

[6] Symes, D., and Ellis, R. 2009. "A Preliminary Study into Rider Asymmetry within Equitation." The Veterinary Journal 181 (1): 34-7.

[7] Dyson, S. 2002. "Poor Performance: Cannot or Will not? The Elite Dressage and Three Day Event Horse." Presented at Conference on Equine Sports Medicine and Science, Essen.

[8] Klimke, R., and Klimke, I. 2006. Walk. In Basic Training of the Young Horse, edited by Diggle, M., and Allen, J. A. London: J. A. Allen \& Co Ltd, 114-6.

[9] Broom, L. 2011. "Balancing Act." British Dressage Magazine 11 (1): 31-2.

[10] Pitts, J. 2011. "Fit to Ride?" British Dressage Magazine 11 (1): 17-8.

[11] Hewett, Z., Ransdell, L., Gao,Y., Petlichkoff, L., and Lucas, S. 2011. "An Examination of the Effectiveness of an 8-Week Bikram Yoga Program of Mindfulness Perceived Stress and Physical Fitness." Journal Exercise Science Fitness (9) 2: 87-92.

[12] Odberg, F., and Bouissou, M. 1999. "The Development of Equestrianism from the Baroque Period to the Present Day and Its Consequences for the Welfare of Horses." Equine Veterinary Journal 28 (1): 26-8.

[13] Weishaupt, M., Weistner, T., Waldern, N., Roepstorrf, R., van Weeren, H., Meyer, H., and Johnston, C. 2006. "Effect of Head and Neck Position on Vertical Ground Reaction Forces and Interlimb Co-ordination in the Dressage Horse Ridden in Walk and Trot on the Treadmill." Equine Exercise Physiology (7) 36: 387-92.

[14] Blokhuis, M., Aronsson, A., Hartmann, E., van Reenan, C., and Keeling, L. 2008. "Assessing the Rider's Seat and Horse's Behaviour: Difficulties and Perspectives." Journal of Applied Animal Welfare Science 11 (1): 191-203.

[15] Chen, C., and Lin, S. 2011. "The Impact of Rope Jumping Exercise on Physical Fitness on Visually Impaired Students." Research in Developmental Disabilities 32 (1): 25-9.

[16] Madengue, S., Seck, D., Bishop, D., Cisse, F., Tsala-Mbala, P., and Ahmaidi, S. 2005. "Are Athletes Able to Self-select Their Optimal Warm Up?" Journal Science Medical Sport 8 (1): 26-34.
[17] Nielson, G., and Anderson, L. 2003. "The Association between High Blood Pressure, Physical Fitness and Body Mass Index in Adolescents." Preventative Medicine 36 (2): 229-324.

[18] Owen, A., O’Donovan, G., and Bird, S. 2008. "Sedentary, Active and Athletic Lifestyles: Right and Left Ventricular Long Axis Diastolic Function." International Journal of Cardiology 127 (1): 112-3.

[19] Lagarde, J., Kelso, J., Peham, C., and Licka, T. 2007. "Coordination Dynamics for the Horse-Rider System." Journal of Motor Behaviour 37 (6): 418-24.

[20] Meyners, E. 2011. Rider Fitness: Body and Brain: 180 Anytime, Anywhere Exercises to Enhance Range of Motion, Motor Control, Reaction Time, Flexibility, Balance and Muscle Memory. London: Trafalgar Square Publishing.

[21] Bray, S., Millen, J., Eidsness, J., and Leuzinger, C. 2005. "The Effects of Leadership Style and Exercise Program Choreography on Enjoyment and Intention to Exercise." Psychology of Sport and Exercise 6 (4): 415-25.

[22] Thorell, L., Lindqvist, S., Bergman, S., Bohlin, G., and Klingberg, T. 2009. "Training and Transfer Effects of Executive Functions in Preschool Children." Developmental Science 12 (1): 969-76.

[23] Ortega, F., Ruiz, J., Hurtig-Wennlof, A., and Sjostrom, M. 2008. "Physically Active Adolescents Are More Likely to Have a Healthier Cardiovascular Fitness Level Independently of Their Adiposity Status. The European Heart Study." Physical Activity and Cardiovascular Fitness in Adolescents 61 (2): 123-9.

[24] Weaver, H., Vichas, D., Strutton, P., and Sorinola, A. 2012. "The Effect of an Exercise Ball on Trunk Muscle Responses to Rapid Limb Movement." Gait and Posture 35 (1): 70-7.

[25] Eyigor, S., Karapolat, H., and Durmaz, B. 2007. "Effects of a Group Based Exercise Programme on Physical Performance, Muscle Strength and Quality of Life in Older Women." Archives of Gerontology and Geriatrics 45 (3): 259-71.

[26] Prichard, I., and Tiggermann, P. 2008. "Relations among Exercise Type, Self-objectification, and Body Image in the Fitness Centre Environment: The Role of Reasons for Exercise." Psychology of Sport and Exercise 6 (9): 855-66. 
The Effects of Traditional Neuromuscular Training and Rider Specific Training on Novice Horse-Rider Position

Appendix A

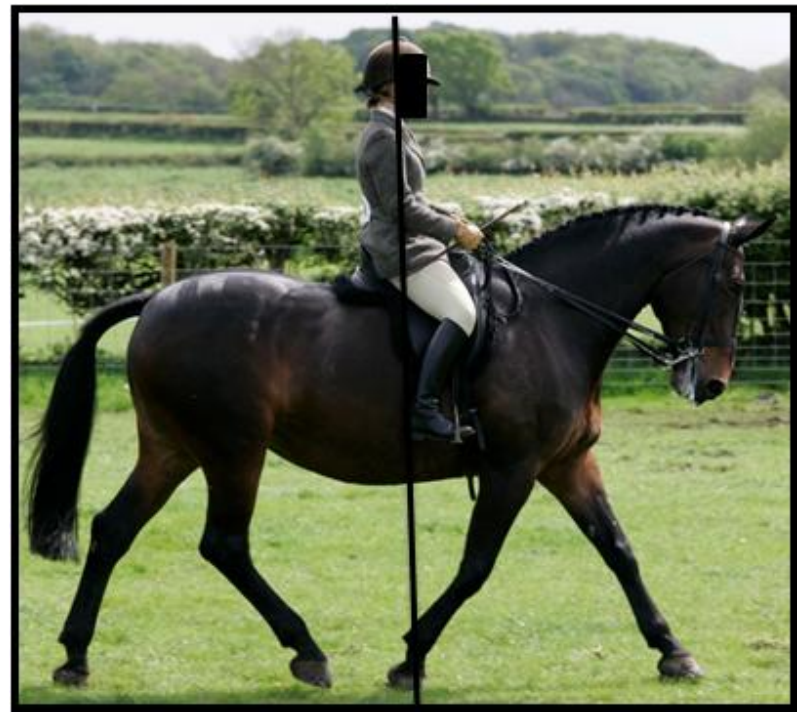

Fig. A1 Rider demonstrating ESHH alignment during the sitting phase of rising trot.

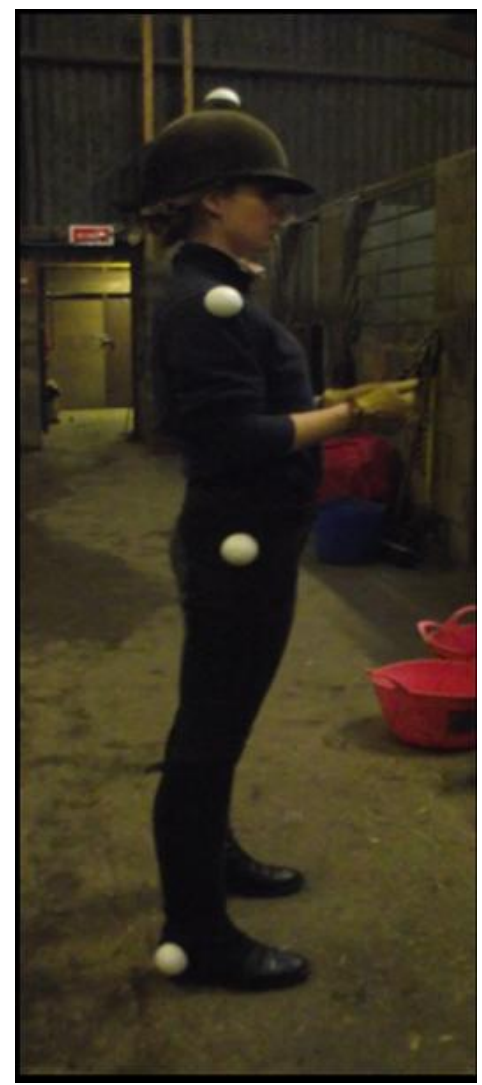

Fig. A2 Marker placement on rider indicating anatomical landmarks to determine ESHH. 